\title{
Benign Biliary Strictures: Narrowing the Differences Between Endoscopic and Surgical Treatments
}

\author{
May Y. W. Wong ${ }^{1} \cdot$ Arthur J. Kaffes $^{1}$
}

Published online: 27 July 2018

(c) Springer Science+Business Media, LLC, part of Springer Nature 2018

Benign biliary strictures (BBS) affecting the common hepatic or bile ducts can be complicated by obstructive jaundice and infection. The majority of benign strictures are iatrogenic, secondary to bile duct injury after liver transplantation or laparoscopic cholecystectomy, with reported incidence ranging from 3 to $13 \%$ [1] and $0.2-0.7 \%$ [2, 3], respectively. They can also be inflammatory, infectious, or complicate diseases such as chronic pancreatitis. Research into preventative measures for patients who are at greater risk of developing BBS such as liver transplant recipients of reduced-size grafts or grafts subjected to prolonged cold ischemic time, or those with hepatic artery thrombosis or blood group $\mathrm{ABO}$ incompatibility $[4,5]$ is needed.

The approach to management is diverse, including endoscopic retrograde cholangiopancreatography and stenting, transhepatic drainage, rendezvous techniques, or by surgical procedures. Nevertheless, these management options are complicated by cholangitis, pancreatitis, bleeding, and perforation. Although endoscopic treatment is regarded as firstline therapy, there is risk of stricture recurrence as well as stent occlusion and migration [6]. Endoscopic management is currently considered first line; the optimal endoscopic intervention for BBS continues to evolve. The authors have previously proposed an endoscopic classification intended to guide treatment based on the location and type of biliary stricture [7].

A meta-analysis of the long-term outcomes of interventions for BBS demonstrated that surgery resulted in the highest long-term stricture resolution rate at $84 \%$, followed by multiple plastic stent insertions at $79 \%$ and covered self-expanding metal stents at $75 \%$, although the differences among these interventions were not significant

Arthur J. Kaffes

arthur@kaffes.com

1 Sydney University Medical School, Royal Prince Alfred Hospital (Director of Endoscopy), Prince of Wales Hospital, Sydney, Australia
[8]. Furthermore, it is costly to manage a BBS; $30 \%$ of patients necessitate multiple hospitalizations and invasive treatment, with a very small percentage of cases progressing to biliary cirrhosis requiring liver transplantation [4]. Therefore, although BBS are not malignant, they are often costly and time-consuming to manage notwithstanding their "benign" designation, requiring timely diagnosis and expert management.

In this issue of Digestive Diseases and Sciences, Li [9] established a novel BBS rabbit model via surgical injury and biliary infection. A biliary infusion tube was inserted into the common bile duct via the stump of cystic duct after cholecystectomy. There were five groups: sham, and biliary infusion with the anti-inflammatory or anti-fibrogenic drugs rapamycin, pirfenidone, or fasudil, or with normal saline. Medications were infused daily during the 4 weeks after surgery. All rabbits formed BBS after surgery: the mortality rate was $13 \%(8 / 60)$, and tube withdrawal rate was $4 \%(2 / 48)$. The authors demonstrated that the thickness of the bile duct wall was significantly decreased, whereas the luminal area of the bile duct was significantly increased in the rapamycin or pirfenidone-treated groups compared to the salinetreated group. Furthermore, the local treatment significantly reduced the expression of proliferation makers, including proliferating cell nuclear antigen (PCNA), collagen I and fibrogenic mediators, including smooth muscle aortic $\alpha-2$ actin (ACTA2) and transforming growth factor (TGF)- $\beta$. Conversely, local treatment ameliorated the expression of inflammatory cytokines such as interleukin (IL)-6, MCP-1, and vascular endothelial growth factor (VEGF). From these results, the authors hypothesized that rapamycin and pirfenidone can prevent BBS formation through anti-proliferative and anti-inflammatory mechanisms, although the effects of fasudil on bile duct area and thickness were less marked.

Over the last decade, there have been only a few experimental and clinical studies focused on the new possibilities for BBS prevention and treatment. Drug-eluting stents in the treatment of biliary tract disease are still at an early 
stage. The benefits of drug-eluting stents in the treatment of luminal disease have been recognized in other areas such as vascular stenosis [10]. Shi [11] infused paclitaxel in a canine model and found it significantly inhibited the activation and proliferation of myofibroblast and excessive collagen deposition in biliary-enteric anastomosis, as well as prohibited scar contracture of anastomotic stoma. Further studies are required to confirm these findings.

The main strengths of the study include the innovative aspect of the in vivo animal model that can serve as a platform for further similar studies. Previously described rabbit models demonstrated notable fibrosis and anastomotic strictures indistinguishable from benign strictures in humans $[12,13]$. Furthermore, the drugs that Li studied have "realworld" applicability; rapamycin is used as an immunosuppressive agent in transplant patients, while pirfenidone is US Food and Drug Administration (FDA) approved for the treatment for idiopathic pulmonary fibrosis, regardless of the disease severity. These drugs inhibit hepatic, lung, and renal fibrosis.

Some methodological flaws that warrant mentioning include the use of a model that has not been validated in prior studies. The study also lacked measurement of hepatic function through biochemical markers such as alanine aminotransferase (ALT), aspartate aminotransferase (AST), and bilirubin. Furthermore, it lacked cholangiographic confirmation of biliary stricture. Postoperative complications rates were high with $53 \%$ of animals having weight loss, abdominal infection, and incision infection. Furthermore, the relatively short duration of the model (28 days) was brief in comparison with clinical postoperative BBS, which usually occur after a few months [5]. The previous rabbit models have used a 6-month follow-up period [13]. Although recognizing this is a pilot study, the small numbers limit the ability to generalize the results.

Future directions include developing studies that could investigate the synergistic effect of these drugs. Furthermore, these studies serve as prelude to the development of effective drug delivery such as via drug-eluting stents which may revolutionize the management of BBS.

In summary, Li's study opens new avenues for exploring the use of pharmacological therapies in future studies, particularly in patients who are high risk of developing BBS.
The optimal endoscopic intervention for BBS continues to progress with an improved understanding of disease pathophysiology. The contribution of additional treatments such as drug-eluting stents could offer a management option that could surpass surgery in efficacy while offering superior patient outcomes.

\section{References}

1. Pascher A, Neuhaus P. Bile duct complications after liver transplantation. Transpl Int Off J Eur Soc Organ Transplant. 2005; 18:627-642.

2. MacFadyen BV Jr, Vecchio R, Ricardo AE, Mathis CR. Bile duct injury after laparoscopic cholecystectomy. The United States experience. Surg Endosc. 1998;12:315-321.

3. Roslyn JJ, Binns GS, Hughes EF, Saunders-Kirkwood K, Zinner MJ, Cates JA. Open cholecystectomy. A contemporary analysis of 42,474 patients. Ann Surg. 1993;218:129-137.

4. Moser MA, Wall WJ. Management of biliary problems after liver transplantation. Liver Transplant Off Publ Am Assoc Study Liver Dis Int Liver Transplant Soc. 2001;7:S46-52.

5. Qian YB, Liu CL, Lo CM, Fan ST. Risk factors for biliary complications after liver transplantation. Arch Surg (Chicago, Ill: 1960). 2004;139:1101-1105.

6. Hu B, Sun B, Cai Q, et al. Asia-Pacific consensus guidelines for endoscopic management of benign biliary strictures. Gastrointestinal Endosc. 2017;86:44-58.

7. Kaffes AJ. Management of benign biliary strictures: current status and perspective. J Hepato Biliary Pancreat Sci. 2015;22:657-663.

8. Huszar O, Kokas B, Matrai P, et al. Meta-analysis of the long term success rate of different interventions in benign biliary stricture. PloS one. 2017;12:e0169618.

9. Yang Q, Wang J, Liu F, et al. A novel rabbit model for benign biliary stricture formation and the effects of medication infusions on stricture formation. Dig Dis Sci. (Epub ahead of print). https ://doi.org/10.1007/s10620-018-5118-0.

10. James SK, Stenestrand U, Lindback J, et al. Long-term safety and efficacy of drug-eluting versus bare-metal stents in Sweden. New Engl J Med. 2009;360:1933-1945.

11. Shi J, Lv Y, Yu L, et al. Interest of a new biodegradable stent coated with paclitaxel on anastomotic wound healing after biliary reconstruction. Eur J Gastroenterol Hepatol. 2013;25:1415-1423.

12. Marubashi S, Dono K, Nagano H, et al. Biliary reconstruction in living donor liver transplantation: technical invention and risk factor analysis for anastomotic stricture. Transplantation. 2009;88:1123-1130.

13. Tian YH, Xu Z, Zhang XQ, Shen W, Zhou XS, Dong JH. Development of an animal model for assessment of primary end-to-end biliary reconstruction. Eur Surg. 2012;44:177-183. 